\title{
Suíno como modelo experimental na pesquisa biomédica: valores fisiológicos normais*
}

\author{
Swine in biomedical research: normal physiological values \\ Giordano Cabral Gianotti', Wanessa Krüger Beheregaray', Simone Passos Bianchi², \\ Verônica Santos Mombach ${ }^{2}$, Adriano Bonfim Carregaro ${ }^{3}$ \& Emerson Antonio Contesini ${ }^{4}$
}

\begin{abstract}
Background: The use of animals in biomedical research is of great importance. It is fundamental for the development and improvement of new practices and technologies. The selection of the species must take into consideration the practicality of handling and the suitability for the experiment, and it must have low operational costs. Swine are important as an experimental model in several experiment areas, because the functionality of many of their organic systems is developed similarly as it is in humans. Swine are animals that become easily stressed. For that reason, their conditioning is necessary to allow the obtainment of data that is closer to normal parameters. This study aims at reporting the physiological values obtained from awake swine, under normal conditions and minimum stress.

Materials, Methods \& Results: The animals were maintained under ideal environmental conditions, and they were always handled and conditioned by the same persons. Twenty crossbred swine were evaluated, aged between 60 and 90 days and with a body mass ranging between 17 and $25 \mathrm{~kg}$. The parameters considered were cardiac frequency (FC), hemoglobin oxygen saturation (pulse oxymetry $-\mathrm{SPO}_{2}$ ), respiratory frequency (fR), end tidal carbon dioxide $\left(\mathrm{ETCO}_{2}\right)$, systolic $(\mathrm{PAM})$, diastolic $(\mathrm{PAD})$ and mean $(\mathrm{PAM})$ arterial pressure and arterial blood gas values $-\mathrm{pH}$, arterial pressure of $\mathrm{CO}_{2}\left(\mathrm{PACO}_{2}\right)$, arterial pressure of $\mathrm{O}_{2}\left(\mathrm{PAO}_{2}\right)$, total $\mathrm{CO}_{2}\left(\mathrm{TCO}_{2}\right)$, base excess $(\mathrm{EB})$, bicarbonate $\left(\mathrm{HCO}_{3}\right)$ and $\mathrm{O}_{2}$ saturation $\left(\mathrm{SATO}_{2}\right)$. The experiment was developed in compliance with the Standards of Animal Ethics and Welfare recommended by the National Council on the Control of Animal Experiments and with Law 11.794 of October 2008, being further approved by the Ethics Committee of the Hospital de Clínicas de Porto Alegre. To obtain the FC, fR, $\mathrm{ETCO}_{2}$ and $\mathrm{SPO}_{2}$ values, 20 animal samples were used. FC presented mean values ( \pm standard deviation) of $134 \pm 20.67$ beats per minute (bpm), showing a maximum value of $180 \mathrm{bpm}$ and a minimum of $103 \mathrm{bpm}$. The mean fR was $24.5 \pm 5.61$ breaths per minute (bpm), with a maximum value of $34 \mathrm{bpm}$ and a minimum of $14 \mathrm{bpm}$, and $\mathrm{SPO}_{2}$ was $96 \pm 2.10 \%$. Moreover, the values obtained in the analysis of exhaled gases showed a $\mathrm{ETCO}_{2}$ at $44.6 \pm 5.41 \mathrm{mmHg}$, with a maximum value of $53 \mathrm{mmHg}$ and minimum value of $34 \mathrm{mmHg}$. The arterial pressure was obtained from 19 animals, yielding the following values: $135.8 \pm 16.29 \mathrm{mmHg}$ for systolic pressure, $81.6 \pm 14.10 \mathrm{mmHg}$ for diastolic pressure and $103.3 \pm 12.65 \mathrm{mmHg}$ for mean pressure. Samples from 18 animals were used to measure the arterial blood gas. The values obtained were as follows: $7.497 \pm 0.019$ for Mean $\mathrm{pH}, 41.7 \pm 6.95 \mathrm{mmHg}$ for partial $\mathrm{O}_{2}$ pressure, $90.65 \pm 2.5$ $\mathrm{mmHg}$ for partial $\mathrm{CO}_{2}$ pressure, $32.8 \pm 2.18 \mathrm{mmol} / \mathrm{L}$ for total $\mathrm{CO}_{2}, 7.57 \pm 1.9 \mathrm{mmol} / \mathrm{L}$ for base excess, $31.5 \pm 2.12 \mathrm{mmol} / \mathrm{L}$ for bicarbonate and $97.55 \pm 0.64$ for oxygen saturation.

Discussion: The animals seemed submissive and able to be conditioned and therefore it was easy to obtain basal values. These data were slightly different from the values obtained in other studies, thus becoming more reliable to be used as parameters in exsperiments employing this species. There seem to be interspecies differences when one intends to make a comparison with human beings. Values such as $\mathrm{O}_{2}$ concentration in arterial blood are normally lower in swine, possibly due to a lower hemoglobin concentration and a higher body temperature in the species. Despite some differences, there are many physiological similarities with human beings, which allows for the conclusion that swine is a great species to be employed in the biomedical research.
\end{abstract}

Keywords: swine, biomedical research, physiological parameters, pigs.

Descritores: suínos, pesquisa biomédica, parâmetros fisiológicos.

*Trabalho financiado pelo Conselho Nacional de Desenvolvimento Científico e Tecnológico (CNPq). ${ }^{1}$ Mestrandos do Programa de Pósgraduação em Ciências Veterinárias (PPGCV), Universidade Federal do Rio Grande do Sul (UFRGS), Av. Bento Gonçalves nº. 9090, Bairro Agronomia, CEP 91540-000 Porto Alegre, RS, Brasil. ${ }^{2}$ Alunos de Graduação, FaVet, UFRGS. ${ }^{3}$ Programa de Pós-graduação em Medicina Veterinária da Universidade Federal da Santa Maria (UFSM), Santa Maria, RS, Brasil. ${ }^{4}$ Departamento de Medicina Animal, FaVet e PPGCV UFRGS. CORRESPONDÊNCIA: G.C. Gianotti [giorgianotti@gmail.com - FAX: + 55 (51) 3316-7305]. 


\section{INTRODUÇÃO}

A escolha da espécie para a experimentação científica é importante, devendo proporcionar praticidade na manipulação, enquadrar-se no ensaio a ser feito e refletir em baixo custo operacional [7]. O suíno é o modelo experimental de escolha para simular diferentes situações médicas, tais como sistemas cardiovascular, tegumentar, urinário e digestivo. Além disso, existe a indicação para estudos de diabetes, toxicologia e choque, sendo ainda considerado como um dos modelos mais adequados para treinamento em cirurgias, laparoscopias, endoscopias e transplantes $[1,8,10]$.

O uso de suínos como modelo clínico em pesquisa vem crescendo desde os anos 1980. É uma espécie única por apresentar características anatômicas e fisiológicas semelhantes a muitos órgãos e sistemas de outras espécies [8]. Por outro lado, devese notar o rápido desenvolvimento físico desses animais desde o nascimento, o que faz com que atinjam em alguns meses, um porte significativo, inviabilizando sua manipulação. Isso implica que apenas possam ser utilizados animais jovens e em experimentos agudos [6].

Geralmente é feita a contenção medicamentosa desses animais, porque eles apresentam uma predisposição ao estresse [2]. Vale destacar, entretanto, a facilidade de manejo e a docilidade constatadas, sem necessidade de contenção medicamentosa, sempre que os animais são habituados aos tratadores, obtendo-se, nessas condições, um rápido condicionamento $[8,10]$.

Com a crescente demanda dessa espécie para ensaios biomédicos e, portanto, de dados relativos às suas funções fisiológicas, optou-se por utilizá-los neste trabalho, cujo objetivo é relatar os valores fisiológicos obtidos de suínos despertos, sob condições normais e mínimo estresse.

\section{MATERIAIS E MÉTODOS}

Neste experimento, foram utilizados 20 suínos mestiços (Sus scrofa domestica), 10 fêmeas e 10 machos castrados, provenientes do Setor de Suinocultura da Faculdade de Veterinária, Universidade Federal do Rio Grande do Sul (UFRGS). Após o desmame, aos 21 dias, os animais foram alocados, em grupos de 6 ou 7, em baias coletivas de 8 metros quadrados, no Hospital de Clínicas Veterinárias (HCV) da UFRGS. Permaneciam em camas de casca de arroz e em condições homeotérmicas entre $20^{\circ} \mathrm{C}$ e $25^{\circ} \mathrm{C}$. Eram alimentados com ração crescimento e água ad libitum. Ao menos três vezes ao dia, eram manipulados por cerca de cinco minutos, treinandoos para os procedimentos, somente por indivíduos integrantes do projeto de pesquisa. Nessas ocasiões, era realizada a limpeza e o fornecimento de complemento alimentar. Evitavam-se sempre estímulos externos e a manipulação dos animais por pessoas estranhas aos mesmos.

Atingindo uma massa corporal entre 17 e 25 $\mathrm{kg}$ (aproxidamente em 90 dias), os animais eram encaminhados à Unidade de Experimentação Animal do Hospital de Clínicas de Porto Alegre (HCPA), dois dias antes das mensurações, e mantidos em condições semelhantes às anteriores. No dia das mensurações, os suínos eram alocados em um carro de transporte manual especialmente adaptado para se obter mínimas situações de estresse. Após passar cerca de uma hora na sala de instrumentação para adaptação ao ambiente, os indivíduos eram sedados. Recebiam isoflurano ${ }^{\mathrm{a}}$ em oxigênio $100 \%$ por vaporizador universal através de máscara, até a completa sedação, sem resposta a estímulos externos. Então, era realizada punção percutânea da veia marginal da orelha, apenas como acesso de segurança, e da artéria plantar medial como acesso para hemogasometria e mensuração da pressão invasiva. Esperava-se a recuperação dos animais. Cerca de 30 minutos depois, quando o analisador de gases indicava ausência de isoflurano na exalação do sistema respiratório e com o suíno completamente responsivo e desperto, podia-se iniciar a avaliação.

Valores como a frequência cardíaca (FC) e saturação de oxigênio em hemoglobina (oximetria de pulso $-\mathrm{SpO}_{2}$ ) eram obtidos através do sensor de oximetria de pulso, fixados na cauda do animal. Somente eram considerados como valores fidedignos aqueles obtidos no monitor, quando o sensor apresentava boa receptividade e interpretação do pulso. Frequência respiratória (fR) e fração expirada de dióxido de carbono $\left(\mathrm{E}_{\mathrm{T}} \mathrm{CO}_{2}\right)$ eram obtidos por meio do sensor nasal conectado ao analisador de gases e aparelho de espirometria. Ainda, no mesmo monitor, eram obtidos os valores de pressão arterial sistólica (PAS), diastólica (PAD) e média (PAM), através do 
sensor de pressão invasiva. Todos os valores eram obtidos mediante um mesmo monitor multiparamétrico ${ }^{\mathrm{b}}$.

A hemogasometria arterial foi realizada no Laboratório de Patologia Clínica do HCPA. O sangue arterial era colhido da artéria plantar medial por meio de seringa heparinizada, e imediatamente encaminhado ao laboratório para análise no hemogasômetro. Este fornecia valores de $\mathrm{pH}$, de pressão arterial de $\mathrm{CO}_{2}\left(\mathrm{PaCO}_{2}\right)$, de pressão arterial de $\mathrm{O}_{2}\left(\mathrm{PaO}_{2}\right)$, de total de $\mathrm{CO}_{2}\left(\mathrm{TCO}_{2}\right)$, de excesso de bases (EB), de bicarbonato $\left(\mathrm{HCO}_{3}\right)$ e de saturação $\mathrm{O}_{2}$ em sangue arterial $\left(\mathrm{SatO}_{2}\right)$.

Todas as avaliações e mensurações foram realizadas com o animal em estação e em condições ambientais de temperatura a $20^{\circ} \mathrm{C}$, a 1 atm com estímulos externos e fatores estressantes mínimos.

A metodologia aplicada para a consecução dos objetivos foi a análise descritiva através de média e desvio padrão ao nível de confiança de 0,95 $(\mathrm{p} \leq 0,05)$. As análises foram efetuadas por meio do programa estatístico SPSS (Statistical Package for Social Science - versão 16.0) $)^{\mathrm{c}}$.

Cabe ainda registrar que o trabalho foi desenvolvido atendendo os padrões éticos e de bemestar animal recomendados pelo CONCEA (Conselho Nacional de Controle de Experimentação Animal) e atendendo ao que está na Lei 11.794 de outubro de 2008. O trabalho foi desenvolvido somente após a aprovação do Comitê de Ética do HCPA, sob registro 08619.

\section{RESULTADOS}

Após a coleta e análise de dados e submissão destes aos intervalos de confiança, optou-se também por relatá-los em caráter de valores máximos e mínimos. Houve casos de animais eliminados do experimento por dificuldade na coleta dos dados: o acesso arterial devia ser padrão, isto é, sempre na mesma região anatômica. Quando isso não era possível, o animal era eliminado do experimento para o procedimento não diferir dos demais.

Para a obtenção de valores de $\mathrm{FC}, \mathrm{fR}, \mathrm{E}_{\mathrm{T}} \mathrm{CO}_{2}$ e $\mathrm{SpO}_{2}$, foram utilizadas amostras de 20 animais. A FC apresentou valores médios \pm DP (desvio padrão) $134 \pm 20,67$ batimentos por minuto (bpm), com valor máximo de 180 bpm e mínimo de 103 bpm. A fR média \pm DP foi de 24,5 \pm 5,61 movimentos por minuto (mpm), com valor máximo de $34 \mathrm{mpm}$ e mínimo de 14 mpm e $\mathrm{SpO}_{2}$ foi de $96 \pm 2,10 \%$. Ainda, os valores obtidos na análise de gases expirados mostraram $\mathrm{ETCO}_{2}$ em 44,6 $\pm 5,41 \mathrm{mmHg}$, com valor máximo de $53 \mathrm{mmHg}$ e valor mínimo de $34 \mathrm{mmHg}$. As pressões arteriais foram obtidas de 19 animais e registraram 135,8 $\pm 16,29 \mathrm{mmHg}$ para a sistólica, $81,6 \pm 14,10 \mathrm{mmHg}$ para a diastólica e 103,3 $\pm 12,65$ mmHg para a média. A Tabela 1 descreve os respectivos valores.

Tabela 1. Parâmetros fisiológicos normais \pm DP (desvio padrão) - Frequência Cardíaca (FC), Oximetria $\left(\mathrm{SpO}_{2}\right)$, Frequência Respiratória (fR), Fração Expirada de $\mathrm{CO}_{2}(\mathrm{E} \mathrm{TCO})$, Pressão Arterial Sistólica (PAS), Pressão Arterial Diastólica (PAD) e Pressão Arterial Média (PAM) - de suínos mestiços (Sus scrofa domestica) com 90 dias e massa corporal entre 17 e $25 \mathrm{~kg}$.

\begin{tabular}{ccccc}
\hline Variável & Média* $^{*}$ & Valor Máximo & Valor Mínimo & $\mathrm{n}$ \\
\hline $\mathrm{FC}(\mathrm{bpm})$ & $134 \pm 20,67$ & 180 & 103 & 20 \\
$\mathrm{SpO}_{2}(\%)$ & $96 \pm 2,10$ & 100 & 92 & 20 \\
$\mathrm{fR}(\mathrm{mpm})$ & $24,5 \pm 5,61$ & 34 & 14 & 20 \\
$\mathrm{E}_{\mathrm{T}} \mathrm{CO}_{2}(\mathrm{mmHg})$ & $44,6 \pm 5,41$ & 53 & 34 & 20 \\
$\mathrm{PAS}(\mathrm{mmHg})$ & $135,8 \pm 16,29$ & 165 & 108 & 19 \\
$\mathrm{PAD}(\mathrm{mmHG})$ & $81,6 \pm 14,10$ & 106 & 54 & 19 \\
$\mathrm{PAM}(\mathrm{mmHg})$ & $103,3 \pm 12,65$ & 129 & 80 & 19 \\
\hline
\end{tabular}


Para obtenção dos valores de hemogasometria arterial, presentes na Tabela 2, utilizou-se amostras de 18 animais. Os valores médios $\pm \mathrm{DP}$ do $\mathrm{pH}$ foram $7,497 \pm 0,019$; da pressão parcial de $\mathrm{O}_{2}$ foram $41,7 \pm$ $6,95 \mathrm{mmHg}$; da pressão parcial de $\mathrm{CO}_{2}$ foram 90,65 $\pm 2,5 \mathrm{mmHg}$; da pressão total de $\mathrm{CO}_{2}$ foram $32,8 \pm$ $2,18 \mathrm{mmol} / \mathrm{L}$; excesso de bases 7,57 $\pm 1,9 \mathrm{mmol} / \mathrm{L}$; Bicarbonato $31,5 \pm 2,12 \mathrm{mmol} / \mathrm{L}$; e saturação oxigênio de $97,55 \pm 0,64$.

\section{DISCUSSÃO}

Foi perceptível a facilidade de manipulação dos animais e a adaptação dos mesmos à técnica. Essa informação, já descrita anteriormente, demonstra que os animais, quando condicionados, podem transformar-se em excelente modelo experimental para diversas situações. A importância de simular novas situações de condicionamento, minimizando o estresse dos animais, pode resultar em diferenças nas análises dos parâmetros fisiológicos $[1,8]$.

O anestésico é uma droga de escolha na anestesia e sedação da espécie por apresentar poucos efeitos adversos e rápida recuperação dos pacientes. Apesar disso, o isoflurano pode causar hipotensão e depressão respiratória dose dependente. É possível afirmar que a sedação a que foram submetidos os indivíduos não interferiu nos seus dados fisiológicos. Isso deve-se ao fato de a exposição do animal à droga ter durado um pequeno intervalo de tempo, e de a metabolização da droga em nível sistêmico ter sido mínima, com rápida eliminação pela via respiratória. Todos esses fatores foram ainda comprovados pelo analisador de gases, que demonstrava nas mensurações a não eliminação do fármaco pelo paciente $[3,5,10]$.

Nas mensurações realizadas, dados relativos à dinâmica respiratória, comportaram-se de maneira semelhante às relatadas por outros autores. Relatamse fRs entre 16 e $25 \mathrm{mpm}$ em suínos entre 25 e $25 \mathrm{~kg}$ [4]. Em outros casos, de maneira controversa, suínos com pouco menos de $13 \mathrm{~kg}$ mostraram frequências médias de 13mpm [11]. Valores como oximetria e fração expirada de $\mathrm{CO}_{2}$ mantiveram-se em parâmetros aceitáveis e já conhecidos para a espécie, mostrando ser confiáveis.

Os parâmetros cardiocirculatórios não demonstraram grandes variações quando comparados com outros estudos. Os parâmetros FC, PAS, PAD e PAM mantiveram-se nos níveis normais para a espécie [4].

As análises hemogasométricas de sangue arterial mantiveram-se dentro da normalidade. Quando existe a intenção de realizar um comparativo com humanos, parece existir diferenças interespécies. Valores como concentração de $\mathrm{O}_{2}$ em sangue arterial normalmente são mais baixos em suínos, provavelmente por existir menor concentração de hemoglobina na espécie, além de a temperatura corpórea ser

Tabela 2. Parâmetros fisiológicos normais $\pm \mathrm{DP}$ (desvio padrão) $-\mathrm{pH}$, Pressão Arterial de $\mathrm{CO}_{2}$ $\left(\mathrm{PaCO}_{2}\right)$, Pressão Arterial de $\mathrm{O}_{2}\left(\mathrm{PaO}_{2}\right)$, Total de $\mathrm{CO}_{2}\left(\mathrm{TCO}_{2}\right)$, Excesso de Bases $(\mathrm{EB})$, Bicarbonato $\left(\mathrm{HCO}_{3}\right)$ e Saturação $\mathrm{O}_{2}\left(\mathrm{SatO}_{2}\right)$, em gasometria de sangue arterial - de suínos mestiços (Sus scrofa domestica) com 90 dias e massa corporal entre 17 e $25 \mathrm{~kg}$.

\begin{tabular}{ccccc}
\hline Variável & Média & Valor Máximo & Valor Mínimo & $\mathrm{n}$ \\
\hline $\mathrm{pH}$ & $7,497+0,019$ & 7,54 & 7,453 & 18 \\
$\mathrm{PaCO}_{2}(\mathrm{mmHg})$ & $41,7+2,5$ & 44,4 & 35,8 & 18 \\
$\mathrm{PaO}_{2}(\mathrm{mmHg})$ & $90,65+6,95$ & 107,7 & 81,6 & 18 \\
$\mathrm{TCO}_{2}(\mathrm{mEq} / \mathrm{L})$ & $32,8+2,18$ & 38 & 28,9 & 18 \\
$\mathrm{~EB}_{(\mathrm{mEq} / \mathrm{L})}$ & $7,57+1,9$ & 12,6 & 4,6 & 18 \\
$\mathrm{HCO}_{3}(\mathrm{mEq} / \mathrm{L})$ & $31,5+2,12$ & 36,6 & 27,8 & 18 \\
$\mathrm{SatO}_{2}$ & $97,55+0,64$ & 99,6 & 96,8 & 18 \\
\hline
\end{tabular}


mais elevada. Bicarbonato e $\mathrm{pH}$ são sensivelmente mais altos em suínos quando comparados com humanos [4].

\section{CONCLUSÃO}

A maioria dos dados obtidos neste experimento permitiu concluir que, quando comparados com valores anteriormente relatados, eles estão de acordo com o padrão da espécie, podendo fornecer subsídios e atualização para novas pesquisas. É interessante ressaltar que diferentes técnicas para obtenção desses valores podem resultar em discrepâncias fisiológicas em detrimento de situações de menor estresse e condicionamento. Com relação às semelhanças sistêmicas existentes entre seres humanos e suínos, esta extrapolação deve ser criteriosa, pois, em relação aos exames de hemogasometria, as diferenças são significativas. Entretanto, a espécie estudada apresenta um perfil adequado para pesquisa biomédica.

\section{NOTAS INFORMATIVAS}

a Forane - Cristália Produtos Químicos e Farmacêuticos Ltda., Itapira, SP, Brasil.

${ }^{b}$ Monitor Datex-Ohmeda - General Eletric Healthcare Company, P.O. Box 900, FIN-00031 GE, Finland.

'SPSS (Statistical Package for Social Science) Incorporation - IBM Company, Chicago, EUA.

\section{REFERÊNCIAS}

1. Bay A., Carbone L., Frank W. \& Qumby F. 1995. Swine. In: Rollim B.E. \& Kesel M.L. (Eds). The Experimental Animal in Biomedical Research. Boca Ratón: CRC press inc, p167-194.

2. Flecknell P.A. 2009. Laboratory Animal Anaesthesia. 2nd edn. San Diego: Academic Press Limited, 300p.

3. Hall L.W., Clarke K.W. \& Trim C.M. 2001. Veterinary Anaesthesia. 10th edn. London: Harcourt Publishers Limited, 561p.

4. Hannon P.J., Bossone C.A. \& Wade C.E. 1990. Normal physiological values for concious pigs used in biomedical research. Laboratory Animal Science. 40(3): 293-298.

5. Kaiser G.M., Heuer M.M., Frühauf N.R., Kühne C.A. \& Broelsch C.E. 2006. General Handling and Anesthesia for Experimental Surgery in Pigs. Journal of Surgical Research. 130(1): 73-79.

6. Mariano M. 2003. Minisuíno (minipig) na pesquisa biomédica experimental. Acta Cirúrgica Brasileira. 18(5): 387-391.

7. Massone F. 2003. Anestesiologia Veterinária: Farmacologia e Técnicas. 4.ed. Rio de Janeiro: Guanabara Koogan S.A., 252p.

8. Smith A.C. \& Swindle M.M. 2006. Preparation of swine for the laboratory. Institute for Laboratory Animal Research. 47(4): 358-363.

9. Swindle M.M. 2007. Swine in the Laboratory: Surgery, Anesthesia, Imaging, and Experimental Techniques. 2nd edn. Boca Raton: CRC Press, 496 p.

10. Tranquilli W.J., Thurmon, J.C. \& Grimm K.A. 2007. Veterinary Anesthesia and Analgesia. 4th edn. Iowa: Blackwell Publishing, 1096p.

11. Verbrugghe C., Laurent P. \& Bouvert P. 1982. Chemoreflex drive of ventilation in the awake miniature pig. Respiratory Physiology. 47(3): 379-391. 
